\title{
Disruption of Platelet-derived Chemokine Heteromers Prevents Neutrophil Extravasation in Acute Lung Injury
}

\author{
Jochen Grommes 1,2, Jean-Eric Alard1,3, Maik Drechsler1,3, Sarawuth Wantha1,3, Matthias Mörgelin4, \\ Wolfgang M. Kuebler,6, Michael Jacobs², Philipp von Hundelshausen 1,3,7, Philipp Markart ${ }^{8}$, \\ Malgorzata Wygrecka8, Klaus T. Preissner9, Tilman M. Hackeng7, Rory R. Koenen ${ }^{1,3,7}$, \\ Christian Weber $3,7,10$, and Oliver Soehnlein $1,3,7$
}

\begin{abstract}
${ }^{1}$ Institute for Molecular Cardiovascular Research and ${ }^{2}$ Department of Vascular Surgery, Rheinisch-Westfälische Technische Hochschule, Aachen, Germany; ${ }^{3}$ Institute for Cardiovascular Prevention, Ludwig-Maximilians-University, Munich, Germany; ${ }^{4}$ Division of Infection Medicine, Department of Clinical Sciences, Lund University, Lund, Sweden; ${ }^{5}$ Keenan Research Center, Li Ka Shing Knowledge Institute of St. Michael's Hospital, Toronto, Canada; ${ }^{6}$ Institute of Physiology and German Heart Institute, Berlin, Germany; ${ }^{7}$ Cardiovascular Research Institute Maastricht, Maastricht University, Maastricht, The Netherlands; ${ }^{8}$ Department of Internal Medicine, University of Giessen Lung Center, Giessen, Germany; ${ }^{9}$ Department of Biochemistry, Justus-Liebig-University, Giessen, Germany; and ${ }^{10}$ Munich Heart Alliance, Munich, Germany
\end{abstract}

\begin{abstract}
Rationale: Acute lung injury (ALI) causes high mortality, but its molecular mechanisms and therapeutic options remain ill-defined. Gram-negative bacterial infections are the main cause of ALI, leading to lung neutrophil infiltration, permeability increases, deterioration of gas exchange, and lung damage. Platelets are activated during ALI, but insights into their mechanistic contribution to neutrophil accumulation in the lung are elusive.
\end{abstract}

Objectives: To determine mechanisms of platelet-mediated neutrophil recruitment in ALI.

Methods: Interference with platelet-neutrophil interactions using antagonists to P-selectin and glycoprotein Ilb/IIla or a small peptide antagonist disrupting platelet chemokine heteromer formation in mouse models of ALI.

Measurements and Main Results: In a murine model of LPS-induced ALI, we uncover important roles for neutrophils and platelets in permeability changes and subsequent lung damage. Furthermore, platelet depletion abrogated lung neutrophil infiltration, suggesting a sequential participation of platelets and neutrophils. Whereas antagonists to $P$ selectin and glycoprotein Ilb/Illa had no effects on LPS-mediated ALI, antibodies to the platelet-derived chemokines CCL5 and CXCL4 strongly diminished neutrophil eflux and permeability changes. The two chemokines were found to form heteromers in human and murine ALI samples, positively correlating with leukocyte influx into the lung. Disruption of CCL5-CXCL4 heteromers in LPS-, acid-, and sepsis-induced ALI abolished lung edema, neutrophil infiltration, and tissue damage, thereby revealing a causal contribution.

(Received in original form August 23, 2011; accepted in final form December 29, 2011) Supported by the Deutsche Forschungsgemeinschaft (SO876/3-1, FOR809 TP2 and TP9), the German Heart Foundation, the Else-Kröner-Fresenius Foundation, and the B. Braun Foundation.

Author Contributions: J.G. performed all in vivo experiments and contributed to manuscript preparation; J.-E.A. contributed to bronchoalveolar lavage analyses; M.D. contributed to fluorescence-activated cell sorter analyses; S.W. performed flow chamber analyses; M.M. performed scanning electron microscopy; W.M.K. contributed to intravital imaging and provided intellectual input; M.J. and P.v.H. provided intellectual input; P.M., M.W., and K.T.P. provided human bronchoalveolar lavage samples and analyzed and interpreted the data; T.M.H. synthetized MKEY; R.R.K. provided intellectual input, performed heteromer analyses, and contributed to writing; C.W. provided intellectual input and funding; and O.S. designed the study, provided funding, and wrote the manuscript.

Correspondence and requests for reprints should be addressed to Oliver Soehnlein, M.D., Ph.D., Institute for Cardiovascular Prevention, Ludwig-Maximilians-University Munich, Pettenkoferstrasse 9, 80336 Munich, Germany. E-mail oliver.soehnlein@med. uni-muenchen.de

This article has an online supplement, which is accessible from this issue's table of contents at www.atsjournals.org

Am J Respir Crit Care Med Vol 185, Iss. 6, pp 628-636, Mar 15, 2012

Copyright $\odot 2012$ by the American Thoracic Society

Originally Published in Press as DOI: 10.1164/rccm.201108-15330C on January 12, 2012 Internet address: www.atsjournals.org

\section{AT A GLANCE COMMENTARY}

Scientific Knowledge on the Subject

Neutrophils emigrating from the microcirculation prominently contribute to acute lung injury. Activation of platelets facilitates neutrophil extravasation by various mechanisms.

What This Study Adds to the Field

We unveil an important contribution of platelet chemokine heteromers to neutrophil recruitment in acute lung injury. Disruption of heteromer formation is here shown to largely diminish neutrophil extravasation.

Conclusions: Taken together, our data identify a novel function of platelet-derived chemokine heteromers during ALI and demonstrate means for therapeutic interference.

Keywords: neutrophil; platelet; chemokine; recruitment; acute lung injury

Acute lung injury (ALI) is a life-threatening disease with an ageadjusted incidence of 86.2 per 100,000 person-years (1). Despite innovations in intensive care medicine, the mortality of ALI remains approximately $40 \%$. ALI is characterized by an increased permeability of the alveolar-capillary barrier, resulting in lung edema with protein-rich fluid and consequently in impaired arterial oxygenation. A major cause for development of ALI is sepsis, wherein gram-negative bacteria are the dominating factor. LPS inhalation mimics human gram-negative ALI, leading to recruitment of neutrophils, pulmonary edema, and finally impairment of gas exchange (2).

Recruitment of neutrophils is a key event in development of ALI (3) resulting in plasma leakage and deterioration of oxygenation. The importance of neutrophils in ALI is supported by studies, where lung injury was abolished or reversed by depletion of neutrophils $(4,5)$. Much of the neutrophil-dependent ALI is thought to be mediated by granule proteins released from activated neutrophils. For example, azurocidin and $\alpha$-defensins have been found to directly affect permeability changes $(6,7)$, whereas proteases of neutrophilic origin, such as neutrophil elastase, have been implicated in the degradation of surfactant proteins, epithelial cell apoptosis, and coagulation $(8,9)$. Under inflammatory conditions, platelets prominently interact with neutrophils, thus promoting their recruitment by such mechanisms as formation of platelet-neutrophil aggregates by employment of PSGL-1 and P-selectin (10) or 
deposition of platelet-derived chemokines, such as CCL5 and CXCL4 (11). Recent studies provide evidence for the significance of platelets in mouse models of acid-induced ALI (12) and transfusion-related ALI (5). These prompted us to systematically investigate the importance of platelet-neutrophil interactions in various models of ALI. Herein, we demonstrate a dominant role of platelet-derived chemokines and their heteromer formation in neutrophil lung infiltration, edema formation, and tissue damage, a finding further translated into a therapeutic approach.

\section{METHODS}

\section{Murine Models of ALI}

ALI in male C57B1/6 mice (Janvier, St Berthevin Cedex, France), 8 weeks of age, was induced by (1) exposure to aerosolized LPS $(500 \mu \mathrm{g} / \mathrm{ml})$ from Salmonella enteritidis (Sigma, Munich, Germany) for 30 minutes; (2) intratracheal injection of $2 \mu \mathrm{l} / \mathrm{g}$ of $0.1 \mathrm{M} \mathrm{HCl}(\mathrm{pH}=1.5)$; or (3) by cecal ligation and puncture (CLP). Alveolar, interstitial, and intravascular neutrophils were analyzed 4 hours later (in options 1 and 2) or 24 hours later (in option 3), as described (13). To assess lung permeability changes, fluorescein isothiocyanate-dextran $(70 \mathrm{kD})$ was administered intravenously 30 minutes before euthanasia and fluorescein isothiocyanate-dextran clearance was calculated to quantify permeability changes. The fluorescence of bronchoalveolar lavage (BAL) supernatant $\left(100 \mu \mathrm{l}\right.$, Fluo $\left._{\mathrm{BAL}}\right)$ and serum $(50 \mu \mathrm{l}$, Fluo Serum $)$ was measured and permeability volume was determined: $\mathrm{V}_{\text {Perm }}=\left[\left(\right.\right.$ Fluo $\left._{\text {BAL }} / 100 \mu \mathrm{l}\right) /\left(\right.$ Fluo $\left.\left._{\text {Serum }} / 50 \mu \mathrm{l}\right)\right] \times \mathrm{V}_{\mathrm{BAL}}$.

Platelets were depleted by intraperitoneal injection of $50 \mu \mathrm{l}$ rabbit antimouse platelet serum (Accurate Chemicals, Westbury, NY). Neutrophils were depleted by intraperitoneal injection of monoclonal antibody 1A8 (100 $\mu \mathrm{g}$; BioXcell). Antibodies to CXCL4 (R\&D Systems, Minneapolis, MN) and CCL5 (eBioscience, San Diego, CA) were injected intraperitoneally (10 $\mu \mathrm{g}$ per mouse) 30 minutes before LPS inhalation. Mice were treated with the peptide antagonist MKEY, designed to specifically disrupt proinflammatory interactions of CCL5-CXCL4 (14), or its scrambled version sMKEY (50 $\mu \mathrm{g}$ per mouse) 12 hours and 1 hour before LPS inhalation or 1 hour after LPS inhalation. All animal experiments were performed after approval by the local authorities for animal experimentation.
A
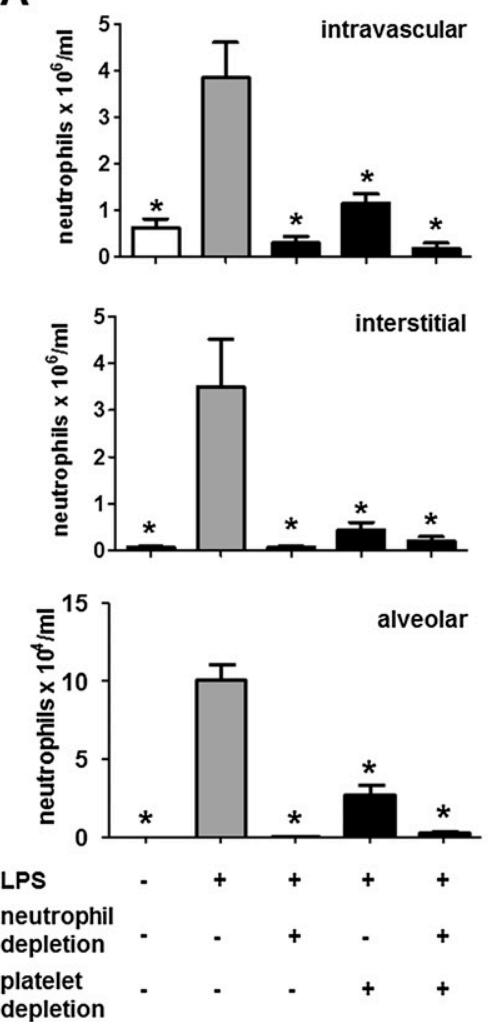

B
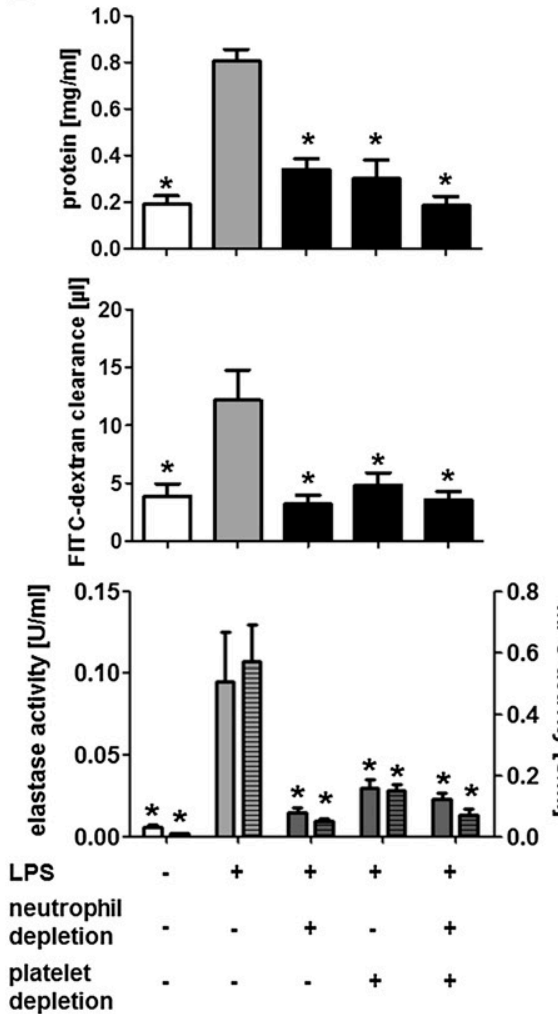

C

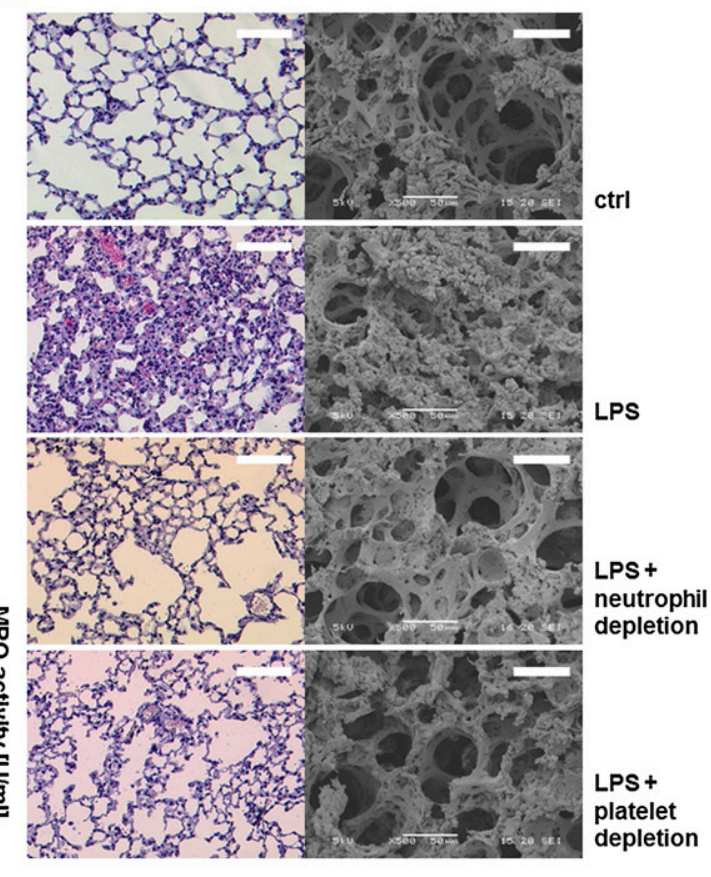

Figure 1. Neutrophils and platelets cooperate in the onset of LPS-induced acute lung injury. Mice were challenged with LPS by inhalation and killed 4 hours later. Neutrophils were depleted by injection of an anti-Ly6G antibody (clone 1A8, $50 \mathrm{ng}$, intraperitoneally), whereas platelets were depleted by application of antiplatelet serum $(50 \mu \mathrm{l}$, intraperitoneally). ( $A$ ) Quantification of intravascular (top), interstitial (middle), and alveolar neutrophils (bottom). (B) Protein concentration (top), fluorescein isothiocyanate (FITC)-dextran clearance (middle), and elastase (bottom, uniform bars) and myeloperoxidase (MPO) activity (bottom, hatched bars) in bronchoalveolar lavage fluids. (C) Representative histologic (left) and scanning electron microscopic (right) images of lungs from mice treated as indicated. Scale bar indicates $50 \mu \mathrm{m}$ for scanning electron microscopy and $250 \mu \mathrm{m}$ for histology. Quantification of histologic lung sections is shown at bottom. $\mathrm{n}=8-10$ for each bar. Statistical significance was tested using one-way analysis of variance with Dunnett post hoc test. *Indicates significant difference compared with LPS-treated animals. 
A
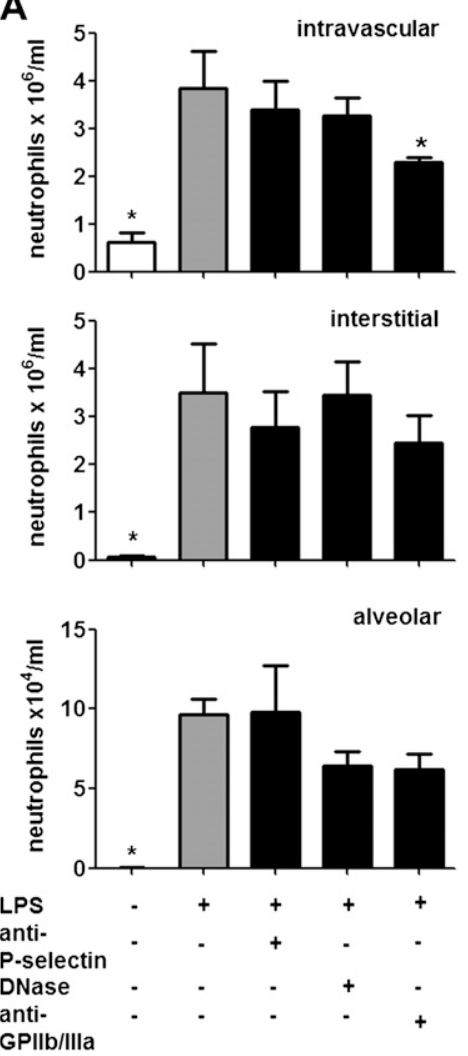

B
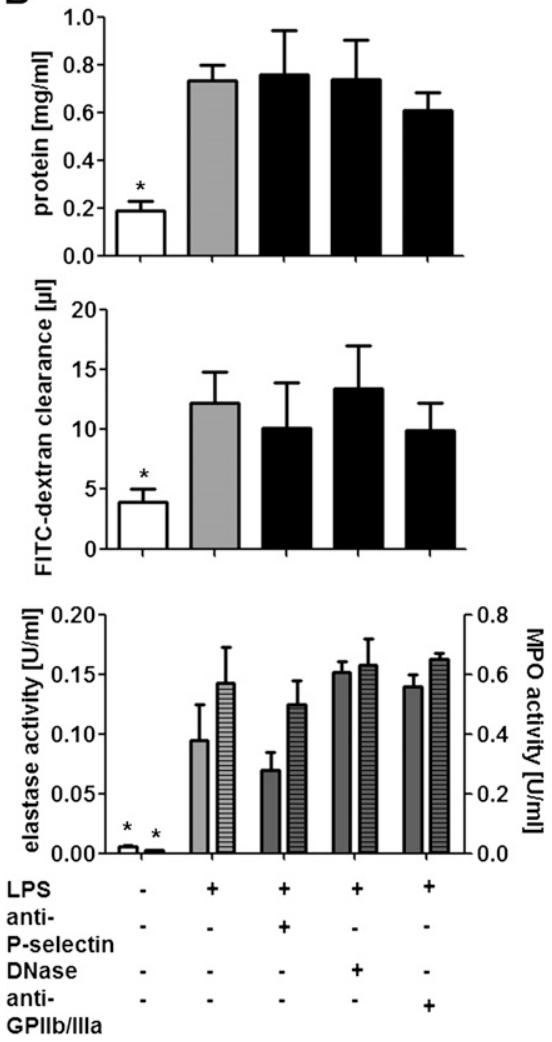

Figure 2. LPS-mediated lung injury is not attenuated by antagonists to P-selectin or by DNase treatment. Mice were treated with antibodies to P-selectin $(30 \mu \mathrm{g})$ or GPIIb/IIla $(100 \mu \mathrm{g})$, or DNase $(1 \mu \mathrm{g})$ before LPS inhalation, and killed 4 hours later. $(A)$ Quantification of intravascular (top), interstitial (middle), and alveolar neutrophils (bottom). (B) Protein concentration (top), fluorescein isothiocyanate (FITC)-dextran clearance (middle), and elastase (bottom, uniform bars) and myeloperoxidase (MPO) activity (bottom, hatched bars) in bronchoalveolar lavage fluids. $\mathrm{n}=8-10$ for each bar. Statistical significance was tested using one-way analysis of variance with Dunnett post hoc test. *Indicates significant difference compared with LPStreated animals.

\section{Histology and Electron Microscopy}

One part of the right lung was fixed in formalin, embedded in paraffin, and stained with Mayer's hematoxylin and eosin for histologic examination. Scoring of histologic sections was done in compliance with the recommendation of the American Thoracic Society (15). See Table E1 in the online supplement for scoring. Another part of the lung was prepared for scanning electron microscopy as described (4).

\section{Intravital Microscopy of the Murine Lung}

After ALI induction, lungs were exposed as described (16). For detection of luminally presented chemokines, Protein G Fluoresbrite YG Microspheres (Polysciences, Eppelheim, Germany) were coupled to polyclonal antibodies to CXCL4 or CCL5 as described (17) and injected intravenously. Antibody and bead complexes were allowed to circulate for 15 minutes and immobilized complexes were detected by intravital microscopy (17).

\section{In Vitro Experimentation}

Neutrophils were incubated with MKEY ( 1 or $10 \mu \mathrm{M})$ for 1 or 3 hours and then activated with $N$-formyl-methionine-leucine-phenylalanine (fMLP) (10 $\mu \mathrm{M}$; Sigma). Up-regulation of $\beta_{1^{-}}$or $\beta_{2}$-integrins was measured after 30 minutes using flow cytometry. To assess neutrophil adhesion, dishes were coated with fibronectin or intercellular adhesion molecule $1(1 \mu \mathrm{g} / \mathrm{ml})$ and neutrophils were perfused at $1 \mathrm{dyne} / \mathrm{cm}^{2}$. Firmly adherent neutrophils were quantified after 4 minutes in multiple fields. Reactive oxygen species (ROS) formation was studied by flow cytometry after neutrophil labeling with $\mathrm{H}_{2}$ DCFDA. To study bacterial killing, neutrophils were incubated with Escherichia coli (D21) for 20 minutes. Thereafter, neutrophils were disrupted by alkaline lysis $\left(\mathrm{H}_{2} \mathrm{O}\right.$ with $\mathrm{NaOH}$ at $\left.\mathrm{pH} 14\right)$. Viable bacteria were grown on Luria Bertani agar overnight and the colonies enumerated. Phagocytosis of $\mathrm{IgG}$ of complement-opsonized fluorescent $E$. coli was analyzed as described (18).

\section{Statistics}

All data are expressed as mean $\pm \mathrm{SD}$. Statistical calculations were performed using GraphPad Prism 5 (GraphPad Software Inc.). Mann-Whitney test, one-way analysis of variance with Dunnett post hoc test, GehanBreslow-Wilcoxon, or Kruskal-Wallis test with post hoc Dunn tests were used as appropriate. Asterisk indicates a $P$ value less than 0.05 .

\section{RESULTS}

\section{Platelet-Neutrophil Interactions Orchestrate Endotoxin-induced Lung Damage}

The involvement of neutrophils as effector cells in ALI is well defined (3). Recently, reports accumulated suggesting the importance of platelets in acid-induced and transfusion-related ALI $(5,12)$. Such data, however, are not available for sepsis models of ALI. Hence, we exposed C57Bl/6 mice to aerosolized LPS and monitored neutrophil recruitment, plasma leakage, lung ultrastructure, and protease activity in the BAL fluid (BALF) (Figure 1, see Figure E1). Such treatment increased the number of intravascular, interstitial, and alveolar neutrophils (Figure 1A) as analyzed by flow cytometry of lung homogenates (see Figure E2) (13). Both the protein concentration and the clearance of fluorescent dextran were found to be increased in the BALF by LPS treatment, indicative of enhanced plasma leakage and edema formation (Figure 1B). Furthermore, the activity of neutrophil-derived elastase and myeloperoxidase was elevated in the BALF of LPS-treated animals (Figure 1B). Histologic and ultrastructural analyses of lungs after LPS exposure revealed alveolar septal thickening, accumulation of inflammatory cells in the interstitium and the alveoli, and influx of protein-rich fluid into the alveolar space compared with control mice exposed to aerosolized saline (Figure 1C).

To assess the individual contribution of neutrophils and platelets to ALI development, each population was depleted individually (see Table E2) (17). Neutrophil depletion abolished alveolar fluid efflux and structural changes confirming the importance of neutrophils in ALI. Moreover, depletion of platelets almost fully abrogated the accumulation of neutrophils in the interstitium and the alveoli, permeability changes, protease release, and structural 

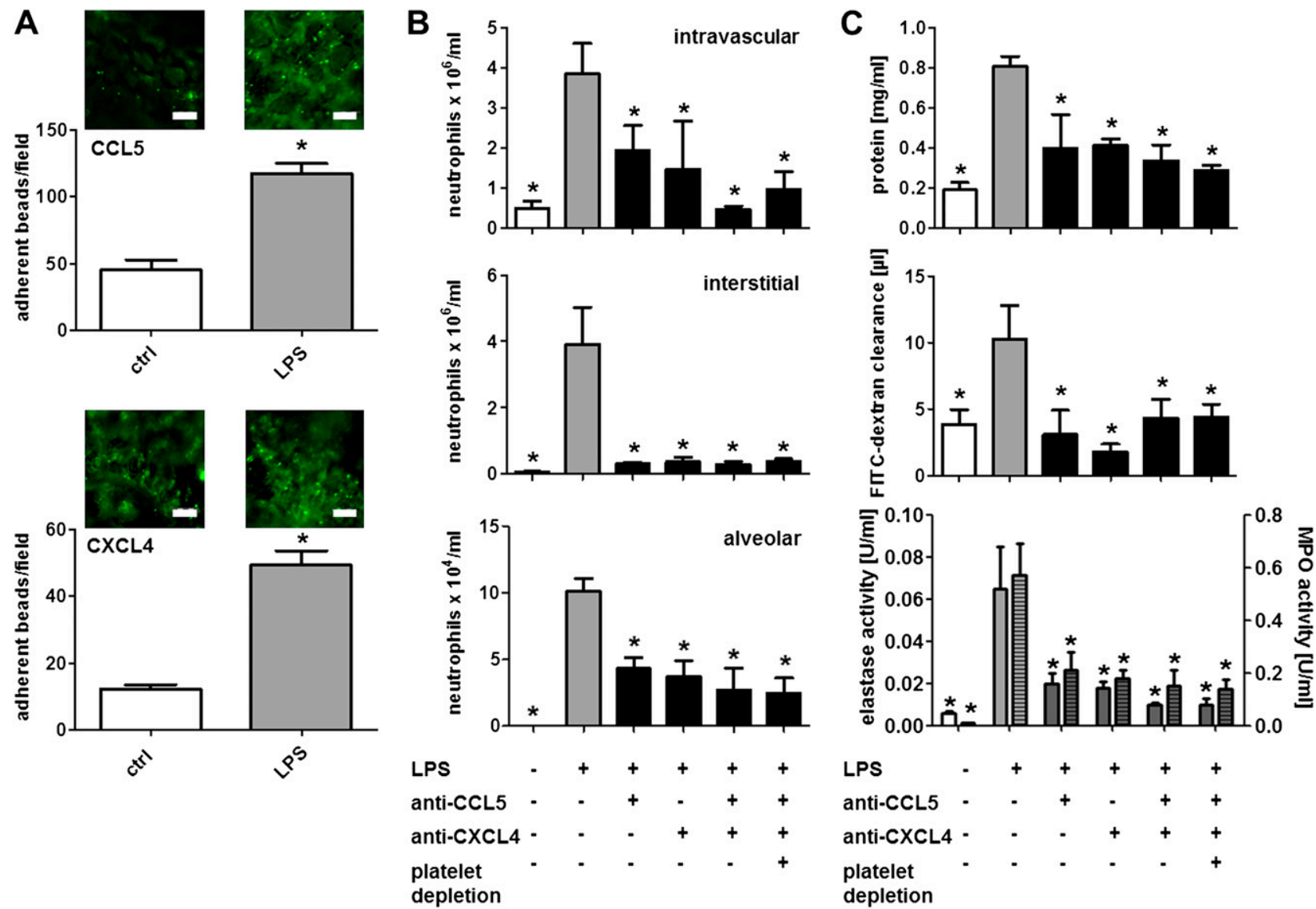

Figure 3. Platelet-derived CCL5 and CXCL4 promote neutrophil recruitment in LPS-induced lung injury. $(A)$ Mice challenged with aerosolized LPS or saline (ctrl) were injected with fluorescent beads conjugated with antibodies to CCL5 (top) or CXCL4 (bottom). Bead immobilization in the lung microcirculation was recorded by fluorescence intravital microscopy. Beads per field were manually counted. Scale bar, $50 \mu \mathrm{m}$. $\mathrm{n}=5$ for each bar. Statistical significance was tested using Mann-Whitney tests. *Indicates significant difference between groups. (B and $C)$ Mice were treated with antibodies to CCL5, CXCL4, or a combination of both. The last group was also depleted of platelets. Thereafter mice were exposed to LPS by inhalation and killed 4 hours later. (B) Displayed are intravascular (top), interstitial (middle), and alveolar neutrophil counts (bottom). (C) Protein concentration (top), fluorescein isothiocyanate (FITC)-dextran clearance (middle), and elastase (bottom, uniform bars) and myeloperoxidase (MPO) activity (bottom, hatched bars) in bronchoalveolar lavage fluids. $\mathrm{n}=8-10$ for each bar. Statistical significance was tested using one-way analysis of variance with Dunnett post hoc test. *Indicates significant difference compared with LPS-treated mice.

changes of the lung tissue (Figures 1A-1C). Finally, we examined whether depletion of both cell subsets would result in an additive effect. However, depletion of neutrophils and platelets together had no such effect (Figures 1A and 1B), suggesting that both cell types act in a sequential context.

\section{No Role of Platelet-Neutrophil Aggregates and Extracellular Nucleotides in LPS-induced ALI}

Both P-selectin and GPIIb/IIIa have been implicated in the formation of platelet-neutrophil complexes and subsequent neutrophil adhesion. To dissect mechanisms underlying the platelet-neutrophil axis dependent lung injury induced by LPS we treated mice with antagonists to P-selectin or an antibody to platelet glycoprotein GPIIb/IIIa before endotoxin inhalation. P-selectin antagonists failed to reduce the intravascular, interstitial, and alveolar accumulation of neutrophils (Figure 2A). Furthermore, inhibition of P-selectin did not affect edema formation and protease release (Figure 2B). Similarly, antibodies to GPIIb/IIIa did not exert effects on alveolar protease activity, plasma leakage, or neutrophil tissue accumulation. However, the intravascular neutrophil counts were significantly reduced by pretreatment with GPIIb/IIIa antibodies (Figure 2).
LPS-mediated activation of platelets stimulates binding of platelets to neutrophils with subsequent release of DNA-containing neutrophil extracellular traps, a mechanism that may be linked to the development of ALI $(9,19)$. In addition, neutrophil extracellular traps release might link to permeability changes as observed in ALI (20). To degrade DNA-containing neutrophil extracellular traps, we injected a bolus of DNase before LPS exposure. However, such treatment failed to reduce LPS-mediated ALI formation (Figure 2).

\section{Platelet-derived Chemokines Are Pivotal in Neutrophil Recruitment in ALI}

As an alternative mechanism, we sought to explore the role of platelet-derived chemokines in neutrophil recruitment and ALI formation. Specifically, platelet-derived CCL5 and CXCL4 were previously shown to mediate monocyte and neutrophil adhesion in large arteries $(11,17)$. Hence, we investigated the deposition of CCL5 and CXCL4 on microvascular lung endothelium by use of intravital microscopy using established protocols $(16,17)$. By this approach we could evidence the increased endothelial presentation of CCL5 and CXCL4 after LPS inhalation (Figure 3A). To further investigate the role of these chemokines in ALI development, we 
A

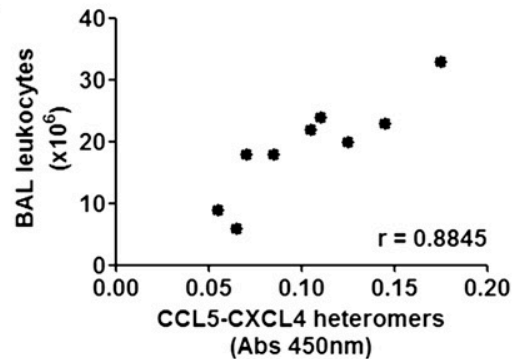

B

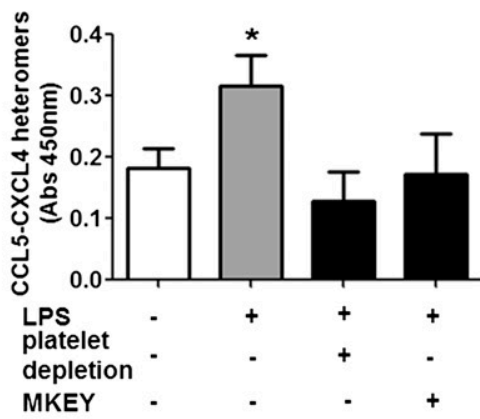

C

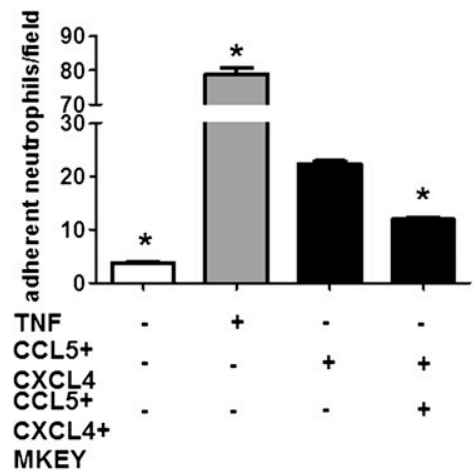

D
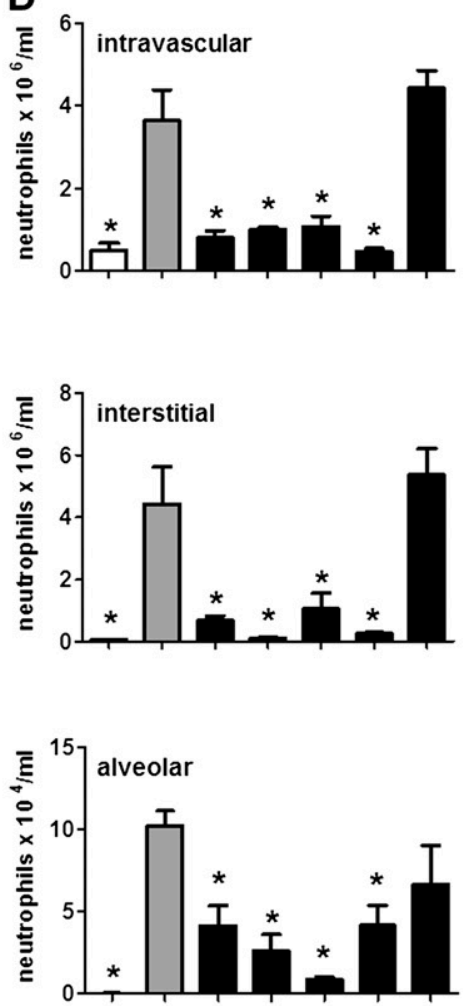

LPS

(before LPS)

MKEY

(after LPS)

anti-CCL5/

anti-CXCL4

SMKEY

platelet

depletion
$\mathbf{F}$

ctrl

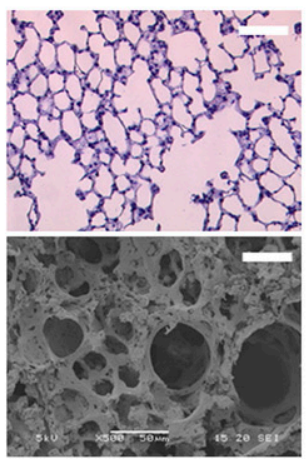

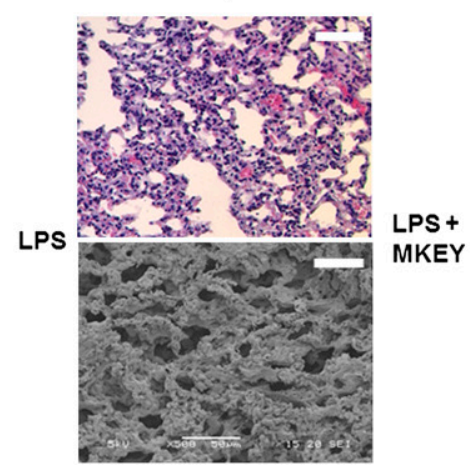

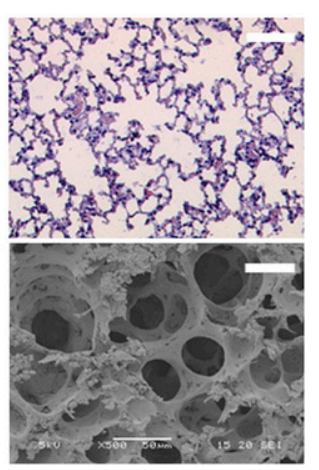

E
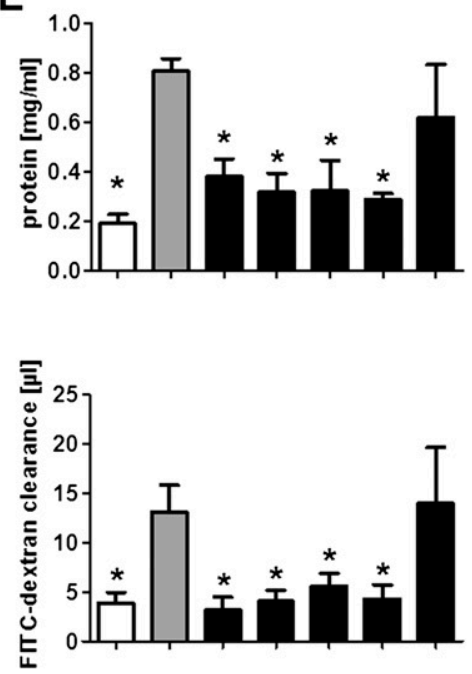

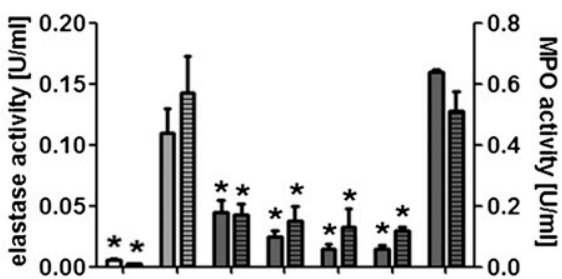

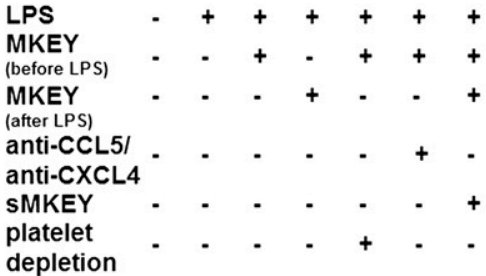

Figure 4. Disruption of CCL5-CXCL4 heteromer formation abrogates neutrophil recruitment in LPS-induced acute lung injury. ( $A$ ) Correlation of leukocyte counts and CCL5-CXCL4 heteromers in bronchoalveolar lavage (BAL) fluid from patients with acute lung injury and adult respiratory distress syndrome. (B) Quantification of CCL5-CXCL4 heteromers in supernatants of homogenates of lungs from mice exposed to LPS and having received antiplatelet serum $(50 \mu \mathrm{l})$ or MKEY $(50 \mu \mathrm{g}) . \mathrm{n}=3$ for each bar. Statistical significance was tested using Kruskal-Wallis test with Dunn post hoc test. *Indicates significant difference to all other groups. (C) Isolated neutrophils were perfused over human umbilical vein endothelial cells (HUVEC) treated with tumor necrosis factor (TNF) $(50 \mathrm{ng} / \mathrm{ml}, 12 \mathrm{~h})$. In addition, recombinant CCL5 and CXCL4 were complexed and immobilized on HUVEC. Thereafter, neutrophils were perfused in presence or absence of MKEY. $n=8$ for each bar. Statistical significance was tested using one-way analysis of variance (ANOVA) with Dunnett post hoc test. *Indicates significant difference compared with HUVEC treated with CCL5-CXCL4 in absence of MKEY. ( $D-F)$ Mice were treated with MKEY (50 $\mu \mathrm{g}) 1$ hour before or after LPS inhalation, scrambled MKEY (SMKEY, $50 \mu \mathrm{g}$ ), antibodies to CCL5 and CXCL4, or platelet-depleting serum. Four hours after LPS inhalation, mice were killed. $(D)$ Displayed are intravascular (top), interstitial (middle), and alveolar neutrophil counts (bottom). (E) Protein concentration (top), fluorescein isothiocyanate (FITC)-dextran clearance (middle), and elastase (bottom, uniform bars) and myeloperoxidase (MPO) activity (bottom, hatched bars) in BAL fluids. $\mathrm{n}=8-10$ for each bar. Statistical significance was tested using one-way ANOVA with Dunnett post hoc test. *Indicates significant difference compared with mice receiving LPS. ( $F$ ) Representative photographs of histologic (left) and scanning electron analyses (right) of mice receiving phosphate-buffered saline (ctrl) or LPS in presence or absence of MKEY. Scale bars indicate $50 \mu \mathrm{m}$ for scanning electron microscopy and $250 \mu \mathrm{m}$ for histology. Quantification of histologic lung sections (bottom). $\mathrm{n}=8-10$ for each bar. Statistical significance was tested using one-way ANOVA with Dunnett post hoc test. *Indicates significant difference compared with LPS-treated animals. 
A
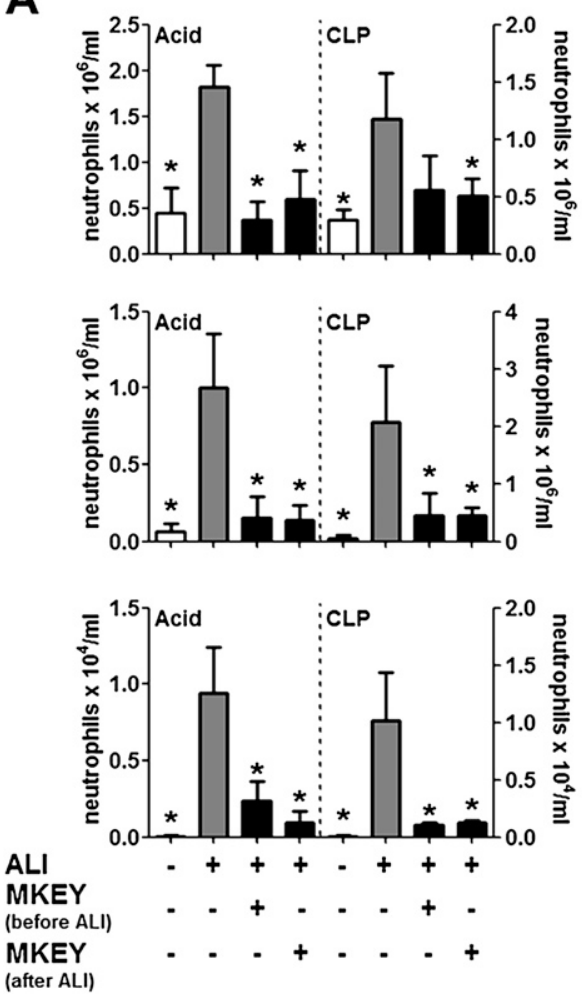

B
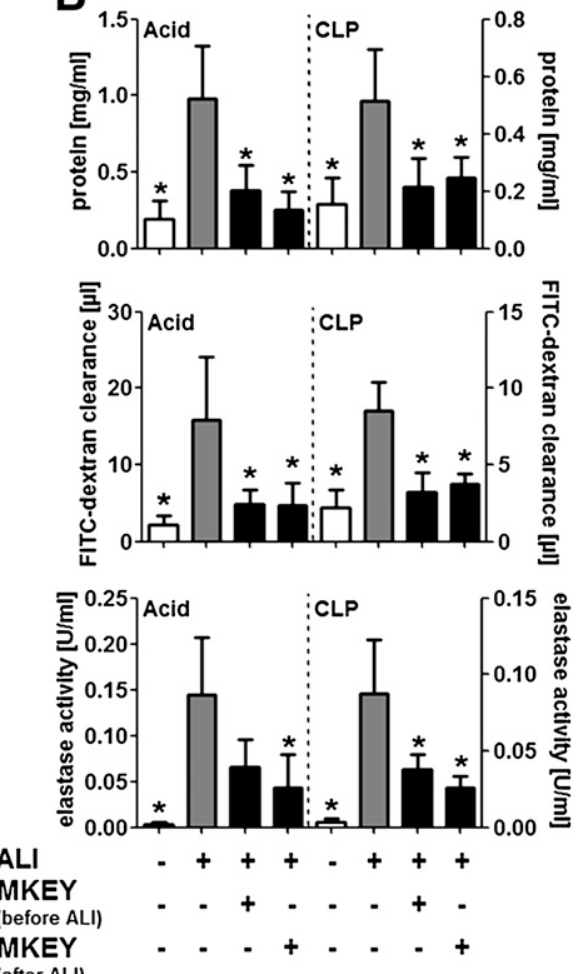

Figure 5. Acid- or sepsis-induced acute lung injury (ALI) is abrogated by disrupting CCL5CXCL4 heteromers. ALI was induced by intratracheal acid application (Acid) or by cecal ligation and puncture (CLP). MKEY $(50 \mu \mathrm{g})$ was injected 1 hour before or 1 hour after ALI induction. Mice were killed 4 (Acid) or 24 hours (CLP) after ALI induction. $(A)$ Displayed are intravascular (top), interstitial (middle), and alveolar neutrophil counts (bottom). (B) Protein concentration (top), fluorescein isothiocyanate (FITC)-dextran clearance ( $\mathrm{mid}$ $d l e$ ), and elastase and myeloperoxidase (MPO) activity (bottom) in bronchoalveolar lavage fluids. $\mathrm{n}=7$ for each bar. Statistical significance was tested using Kruskal-Wallis test with Dunn post hoc test. *Indicates significant difference compared with ALI control mice. treated mice with monoclonal antibodies to CCL5 and CXCL4 individually or in combination before LPS stimulation. Antibodies to CCL5 or CXCL4 significantly reduced neutrophil recruitment in all compartments, plasma exudation, and protease release (Figures $3 \mathrm{~B}$ and $3 \mathrm{C}$ ). Combination of the antibodies and additional depletion of platelets had no additive effects (Figures $3 \mathrm{~B}$ and $3 \mathrm{C}$ ). To further corroborate the role of chemokines originating from bone marrow-derived cells, we reconstituted lethally irradiated mice with bone marrow from $\mathrm{Ccl5}^{-1-}\left(\mathrm{Ccl5}^{-1-} \rightarrow \mathrm{WT}\right)$ or wildtype mice $(\mathrm{WT} \rightarrow \mathrm{WT})$. Mice carrying $\mathrm{Ccl5}^{-/-}$bone marrow exhibited largely decreased neutrophil lung infiltration and plasma leakage in response to LPS compared with mice having received wild-type bone marrow (see Figure E3). Of note, platelet depletion resulted in no further reduction of inflammatory responses in $\mathrm{Ccl5}^{-/-} \rightarrow$ WT mice, whereas neutrophil extravasation and lung edema were thereby reduced in WT $\rightarrow \mathrm{WT}$ mice to levels observed in $\mathrm{Ccl}^{-1-} \rightarrow$ WT mice (see Figure E3). The identification of platelets as a source of CCL5 was further substantiated in experiments where CCL5 was measured in the supernatant of lung homogenates. Although LPS inhalation clearly increased CCL5 levels this was largely reduced in mice depleted of platelets (see Figure E4) but not in mice depleted of neutrophils or monocytes (data not shown).

\section{Platelet Chemokines Form Heteromers, and Their Disruption Prevents LPS-induced ALI}

Heterodimerization of CCL5 and CXCL4 enhances their ability to recruit inflammatory cells $(11,14)$. To analyze the impact of
A
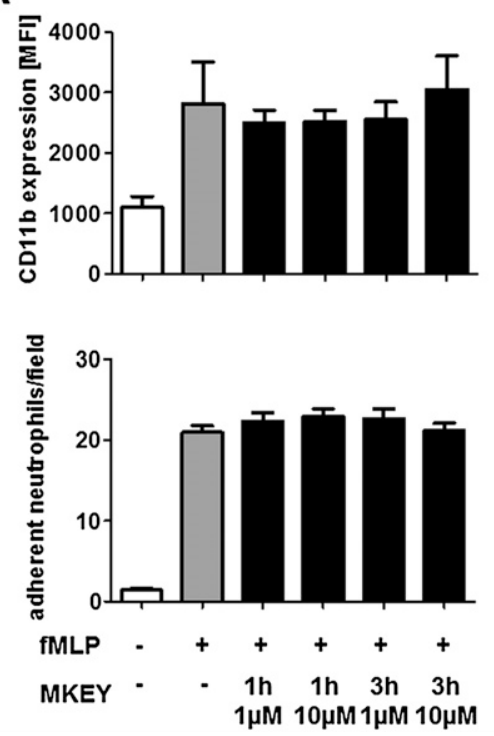

B

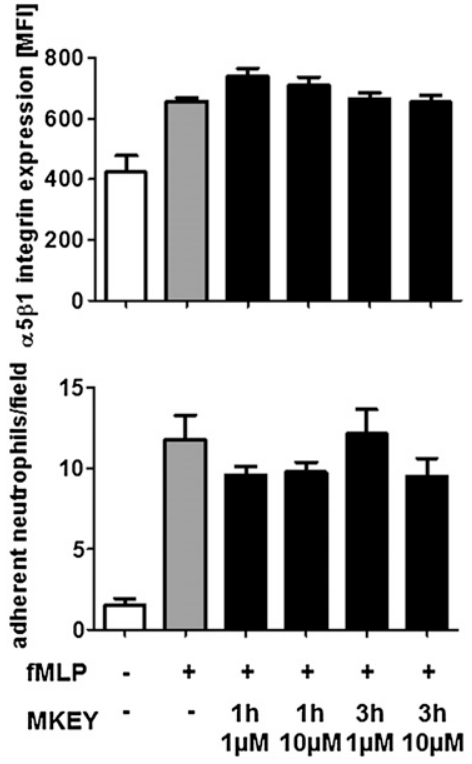

Figure 6. MKEY does not affect neutrophil degranulation and adhesion. Neutrophils were pretreated with MKEY (1 or $3 \mathrm{~h}, 1$ or $10 \mu \mathrm{M}$ ) and then activated with $N$-formylmethionine-leucine-phenylalanine (fMLP). ( $A$ and $B) \mathrm{MFI}$ of surface expression of $\beta_{2}(A, t o p)$ or $\beta_{1}$ integrin ( $B$, top) as measured by fluorescence-activated cell sorter analysis after staining with directly conjugated antibodies. Adhesion of neutrophils perfused over immobilized intercellular adhesion molecule 1 ( $A$, bottom) and fibronectin $(B$, bottom) at $1 \mathrm{dyne} / \mathrm{cm}^{2}$. Number of adherent neutrophils per field is displayed. $\mathrm{n}=3-6$ for fluorescence-activated cell sorter experiments, and 8-10 for flow chamber experiments. Statistical significance was tested using Kruskal-Wallis test with Dunn post hoc test. *Indicates significant difference compared with $\mathrm{fMLP}$ treatment. MFI = mean fluorescence intensity. 
A

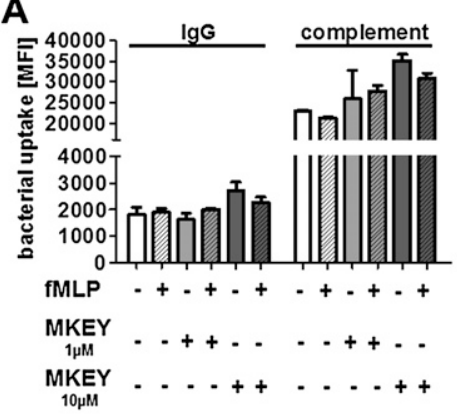

C

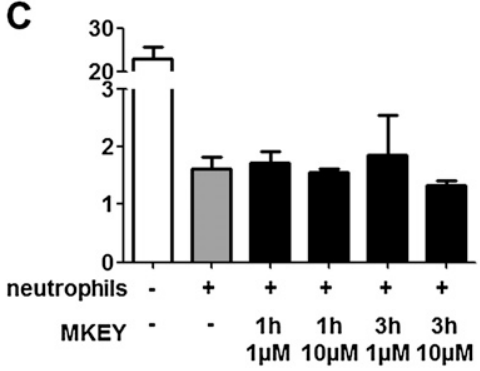

B
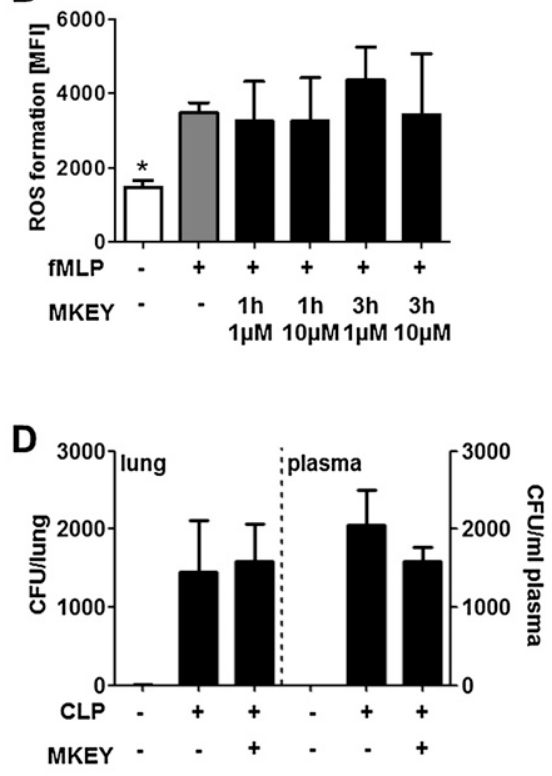

Figure 7. MKEY does not affect neutrophil antimicrobial activity. (A) Neutrophils pretreated with $N$-formyl-methionineleucine-phenylalanine (fMLP) $(10 \mu \mathrm{M}, 15 \mathrm{~min})$ and MKEY ( $3 \mathrm{~h}$ at indicated doses) were incubated with lgG- or complement-opsonized fluorescent Escherichia coli. Uptake was recorded by flow cytometry. $\mathrm{n}=4$ for each bar. (B) Neutrophils were labeled with the reactive oxygen species (ROS)sensitive dye $\mathrm{H}_{2}$ DCFDA and ROS formation was recorded by flow cytometry after fMLP-stimulation in presence or absence of MKEY. Data indicate fluorescence intensity 30 minutes after fMLP exposure. $n=6$ for each bar. Statistical significance was tested using Kruskal-Wallis test with Dunn post hoc test. *Indicates significant difference compared with $\mathrm{MMLP}$ treatment. (C) Formation of colony-forming units (CFU) after hypotonic lysis of neutrophils that had phagocytozed E. coli (D21) for 20 minutes. $\mathrm{n}=5$ for each bar. $(D)$ Bacterial clearance in mice that underwent CLP. Mice received vehicle control or MKEY $(50 \mu \mathrm{g})$ and cultures of lung homogenate or plasma were made on Luria Bertani agar overnight and the colonies were enumerated. $\mathrm{n}=7$ for each bar. MFI = mean fluorescence intensity.
CCL5-CXCL4 heteromers in human ALI and adult respiratory distress syndrome, we analyzed CCL5-CXCL4 heteromers in BALF obtained from patients with severe ALI and adult respiratory distress syndrome (see Table E3). Interestingly, we found a positive correlation between CCL5-CXCL4 heteromers and BALF leukocyte counts (Figure 4A). Furthermore, we quantified the amount of CCL5-CXCL4 heteromers in murine lung homogenates by ELISA. Inhalation of LPS led to a significant formation of heteromers, which was abolished by depletion of platelets (Figure 4B) but not neutrophils (not shown), thus providing evidence for the cellular origin of the chemokine heteromers. We have reported a peptide antagonist, MKEY, designed to specifically disrupt proinflammatory interactions of CCL5-CXCL4, thereby attenuating monocyte recruitment and reducing atherosclerosis without detectable side effects (14). Hence, we treated mice with MKEY before LPS exposure and found that CCL5-CXCL4 formation was fully abrogated (Figure 4B). In addition, in vitro adhesion of neutrophils to CCL5-CXCL4 heteromers deposited on endothelial cells was abolished by presence of MKEY (Figure 4C). Collectively these data indicate the importance of platelet-derived heteromers in lung neutrophil infiltration during ALI and identify possible means for interference.

Next we aimed at investigating the therapeutic potential of disrupting CCL5-CXCL4 interactions. To this end, we treated mice with MKEY before LPS exposure and analyzed lung neutrophils infiltration, edema formation, protease release, and ultrastructural changes (Figures 4D-4F). Treatment with MKEY reduced the number of intravascular neutrophils to baseline levels and significantly reduced alveolar neutrophil counts. In line, lung edema formation was fully abrogated and protease activity was clearly diminished. In contrast, a scrambled version of MKEY (sMKEY) did not exert any of these effects supporting the specificity of MKEY (Figures 4D-4F). Of note, application of MKEY 1 (Figures 4D-4F) or 2 hours (see Figure E5) after LPS inhalation exerted similar beneficial effects, indicating a feasibility of this approach also in therapeutic settings. However, in $\mathrm{Ccl}^{-/-} \rightarrow \mathrm{WT}$ mice MKEY treatment was without effect (see Figure E3) supporting the specificity of this inhibitor. Furthermore, the effects of MKEY treatment matched those observed by platelet depletion or injection of antibodies to CCL5 and CXCL4. Because the latter strategies may result in severe bleeding and adverse effects, such as reduced viral clearance and T-cell proliferation $(21,22)$, interference with CCL5-CXCL4 heteromer formation may stand out as a promising strategy in treating ALI.

\section{Disruption of Heteromers Prevents Acid- and Sepsis-induced ALI}

To test the applicability of MKEY in other models of ALI, we tested its efficacy in models of acid- and sepsis-induced ALI (Figure 5). Intratracheal inoculation of $\mathrm{HCl}$ led to rapid ALI development. Treatment with MKEY before or after instillation of $\mathrm{HCl}$ abrogated neutrophil lung infiltration, edema formation, and discharge of neutrophil elastase (Figure 5). To mimic sepsis, a CLP model was used, thus exposing the mouse to endogenous live bacteria. CLP induced a delayed influx of neutrophils into all three compartments being associated with lung edema formation and neutrophil degranulation (Figure 5). As for the application of LPS and acid, MKEY abrogated CLP-induced neutrophilic lung infiltration, permeability changes, and intraalveolar accumulation of neutrophil elastase (Figure 5), indicative of its broad clinical applicability. This is further supported by Kaplan-Meier analyses, wherein MKEY but not sMKEY improves the survival of mice in the CLP model (see Figure E6).

\section{MKEY Rapidly Distributes to the Lung Microcirculation}

To assess the in vivo behavior of MKEY, we injected a single dose of MKEY intraperitoneally and followed its accumulation in the plasma and the lungs. We observed a rapid increase in plasma levels with a maximal concentration $\left(\mathrm{C}_{\max }\right)$ of $1.21 \mathrm{mg} / \mathrm{ml}$ after 5 minutes (see Figure E7). The concentration of MKEY slowly decreases with a plasma half-life of 3.02 hours and preferentially distributes to the microvasculature of the lungs with a lung/ plasma ratio of 1.5. After 12 hours, MKEY was detectable at a concentration of $14.4 \mathrm{ng} / \mathrm{ml}$ before falling below detection limits after 24 hours.

\section{MKEY Does Not Adversely Affect Neutrophil Effector Functions}

Previous studies have tested the impact of MKEY on macrophagemediated viral clearance, in vivo T-cell function, T-cell proliferation, and macrophage survival and found that none of these functions is 
negatively regulated by MKEY (14). Because neutrophil effector functions, such as emigration, granule mobilization, ROS formation, phagocytosis, and bacterial killing, are vital for a coordinated innate immune response, we tested if any of these functions may be impaired by the presence of MKEY.

For neutrophils to firmly adhere, the up-regulation of $\beta_{2}$-integrins from secretory vesicles is a prerequisite. Such mobilization is mediated by secretagogues, such as the bacterial wall peptide fMLP. Hence, we analyzed the effect of MKEY on fMLP-induced $\beta_{2^{-}}$ integrin up-regulation on neutrophils and their subsequent adhesion to the $\beta_{2}$-integrin ligand intercellular adhesion molecule 1 (Figure 6A). Interestingly, none of these functions was impaired by treatment with MKEY. Because $\beta_{1}$-integrins, however, are crucial for extravascular locomotion of neutrophils, we tested the effect of MKEY on $\alpha_{5} \beta_{1}$-integrin up-regulation and neutrophil adhesion to the $\beta_{1}$-integrin-substrate fibronectin. Again, MKEY did not adversely affect these functions (Figure 6B). In addition, MKEY did not alter transmigration of neutrophils in response to fMLP, keratinocyte-derived chemokine, or macrophage inflammatory protein 2 (see Figure E8). Further to emigration, neutrophils are indispensible in bacterial clearance, much of which is mediated by phagocytosis, production of ROS, and intracellular bacterial killing. To test their phagocytic capacity, fluorescent E. coli bacteria were opsonized with IgG or complement, and incubated with neutrophils treated with fMLP or not in the presence or absence of MKEY. Fluorescence-activated cell sorter analysis revealed no impairment of bacterial uptake by presence of MKEY (Figure 7A). In addition, ROS formation was analyzed after neutrophil labeling with the cell-permeant ROS indicator $\mathrm{H}_{2}$ DCFDA. Presence of MKEY did not alter the fMLP-induced ROS production (Figure 7B). Finally, we assessed the ability of neutrophils to kill E. coli (D21) bacteria after treatment with MKEY. Again, this capacity was not negatively affected by MKEY (Figure 7C). To further test if MKEY would result in impaired host defense we induced sepsis by CLP. Bacterial burden in lungs and plasma was quantified 24 hours after initiation of sepsis (Figure 7D). In these experiments we found no difference between mice treated with MKEY or vehicle control.

\section{DISCUSSION}

Using three independent models of ALI, we here show that neutrophil infiltration into the lung and associated lung damage is driven by platelets. Mechanistically, we identify platelet-derived CCL5 and CXCL4 as crucial mediators in mediating neutrophil lung infiltration. Our previous work has revealed that CCL5 and CXCL4 engage in heterophilic interactions leading to a synergistic enhancement of the cell-recruiting functions of CCL5 $(11,14)$, possibly through an enhancement of CCL5 binding to the leukocyte surface (11). Characterization of the structural determinants of the CCL5-CXCL4 interaction by nuclear magnetic resonance spectroscopy and computational simulations allowed us to design a synthetic peptide, termed MKEY, which was able to specifically disrupt the synergistic interaction between these chemokines by targeting their $\mathrm{N}$-terminal $\beta$-sheet formation (14). We used MKEY as a tool to address the question whether CCL5-CXCL4 heteromers were also involved in the attraction of neutrophils to sites of lung injury. In support of this, we show that the amount of heteromers in human samples positively correlates with the degree of leukocyte influx into the lungs. In addition, abrogation of heteromer formation reduced neutrophil influx into inflamed lungs and subsequent tissue damage. Because this strategy lacked obvious adverse effects on neutrophil antimicrobial activities, we believe that interference with chemokine heteromer formation is a valuable approach to target neutrophil influx into acutely inflamed lungs.
The recruitment of neutrophils is classically defined as a multistep process consisting of leukocyte rolling, activation, adhesion, and subsequent transmigration, involving cell adhesion molecules and chemokines and their respective receptors by the leukocyte recruitment cascade (23). Neutrophil recruitment into the lung is unique and influenced by several factors including neutrophil deformability, adhesion molecules, and the unique capillary structure of the lung (24). On the way through the small pulmonary capillaries, neutrophils have to stop several times to change their shape and subsequently squeeze through the small vessels. Hence, the specific architecture of the lung brings about a prolonged transit time of the neutrophil through the lung and accumulation of neutrophil located primarily within alveolar capillaries. The specific role of adhesion molecules and chemokines in neutrophil recruitment to inflamed lungs is not fully understood and seems to be context-dependent. Although selectins seem to be of minor importance in LPS-induced lung injury, they seem to hold prominent roles in neutrophil recruitment in models of acid-induced lung injury $(12,25)$. In contrast, neutrophil $\beta_{2}$-integrins are of importance in LPS-induced lung inflammation, whereas neutrophil infiltration in acid-induced lung damage occurs independently of CD18 $(26,27)$. Hence, successful targeting of neutrophil emigration in acute lung inflammation is context-dependent and hampered by the complex contribution of various adhesion molecules. In the study provided here, we identify the importance of plateletderived heteromers in neutrophil recruitment to inflamed lungs, and therapeutic disruption of CCL5-CXCL4 heteromers reduces neutrophil lung infiltration independently of the stimulus used, thus suggesting the general importance of this mechanism.

Neutrophil emigration to the lungs is subject to modification by other circulating immune cells including monocytes and platelets $(5,12,28,29)$. Platelets are known to promote neutrophil emigration in various inflammatory models including atherosclerosis (17), kidney failure (30), and ALI $(5,12)$. Mechanisms underlying platelet-mediated neutrophil recruitment include direct cellular interactions involving GPIIbIIIa/Mac-1 and P-selectin/PSGL-1 (31) or neutrophil activation through platelet secretory products (32). In the context of ALI, previous work has focused on direct cellular interactions and provided evidence for the importance of the P-selectin-PSGL-1 axis in acid-induced lung injury (12). In the model of LPS-induced ALI primarily used in the study provided here, no importance of P-selectin or GPIIbIIIa to plateletdependent neutrophil infiltration into the lung was observed. The independency of P-selectin corroborates earlier reports of LPSinduced ALI (29). Hence, we focused on the importance of platelet-derived chemokines with neutrophil-activating capabilities in platelet-mediated neutrophil lung infiltration. Activated platelets release substantial amounts of CCL5 and CXCL4. Because of charge interactions, both chemokines are deposited on endothelial cell surfaces and presented to circulating leukocytes. CCL5 interacts with CCR1, CCR3, and CCR5, whereas CXCL4 was suggested to act through CXCR3. Deficiency in these receptors or the use of antagonists has previously been reported to be associated with diminished neutrophil recruitment in various lung damage models (33-35). In addition, overexpression of human CCL5 in murine lungs increases neutrophil accumulation thus supporting an important role for CCL5 in lung neutrophil recruitment (36). Because CXCL4 alone does not exert classical chemokine functions at submicromolar concentrations (37), its role in cell recruitment might be auxiliary rather than autonomous as was shown for monocytes (38). This is supported by findings that the presence of CXCL4 increased CCL5-induced monocyte arrest on activated endothelium under flow conditions (11). CCL5 and CXCL4 can form a heteromeric complex, which occurs in $\alpha$-granules of human platelets (14). Disruption of such heteromer formation proved effective in treatment of 
atherosclerosis (14) and may hence be an important target in platelet-mediated aggravation of leukocyte recruitment.

Taken together, we provide evidence for the sequential interplay of platelets and neutrophils in LPS-induced ALI. Our data identify heteromer formation of platelet-derived chemokines as a crucial functional link between the two cell types, which was targeted in a therapeutic approach. Indeed, disruption of CCL5-CXCL4 heteromers diminished neutrophil influx, edema formation, and destruction of lung tissue in various in vivo models of ALI. The absence of adverse effects of this strategy would allow for translation toward clinical situations.

Author disclosures are available with the text of this article at www.atsjournals.org

Acknowledgment: The authors acknowledge Xhina Balaj, Silvia Roubrocks, and Sabine Winkler for excellent technical assistance. They also thank Dr. Hannah Nickles for teaching the setup for lung intravital microscopy.

\section{References}

1. Ware LB, Matthay MA. The acute respiratory distress syndrome. $N$ Engl J Med 2000;342:1334-1349.

2. Matute-Bello G, Frevert CW, Martin TR. Animal models of acute lung injury. Am J Physiol Lung Cell Mol Physiol 2008;295:L379-L399.

3. Grommes J, Soehnlein O. Contribution of neutrophils to acute lung injury. Mol Med 2011;17:293-307.

4. Soehnlein O, Oehmcke S, Ma X, Rothfuchs AG, Frithiof R, van Rooijen N, Mörgelin M, Herwald H, Lindbom L. Neutrophil degranulation mediates severe lung damage triggered by streptococcal M1 protein. Eur Respir J 2008;32:405-412.

5. Looney MR, Nguyen JX, Hu Y, Van Ziffle JA, Lowell CA, Matthay MA. Platelet depletion and aspirin treatment protect mice in a twoevent model of transfusion-related acute lung injury. $J$ Clin Invest 2009;119:3450-3461.

6. Gautam N, Olofsson AM, Herwald H, Iversen LF, Lundgren-Akerlund E, Hedqvist P, Arfors KE, Flodgaard H, Lindbom L. Heparin-binding protein (HBP/CAP37): a missing link in neutrophil-evoked alteration of vascular permeability. Nat Med 2001;7:1123-1127.

7. Bdeir K, Higazi AA, Kulikovskaya I, Christofidou-Solomidou M, Vinogradov SA, Allen TC, Idell S, Linzmeier R, Ganz T, Cines DB. Neutrophil alpha-defensins cause lung injury by disrupting the capillary-epithelial barrier. Am J Respir Crit Care Med 2010;181:935-946.

8. Pham CT. Neutrophil serine proteases: specific regulators of inflammation. Nat Rev Immunol 2006;6:541-550.

9. Massberg S, Grahl L, von Bruehl ML, Manukyan D, Pfeiler S, Goosmann C, Brinkmann V, Lorenz M, Bidzhekov K, Khandagale $\mathrm{AB}$, et al. Reciprocal coupling of coagulation and innate immunity via neutrophil serine proteases. Nat Med 2010;16:887-896.

10. Hamburger SA, McEver RP. GMP-140 mediates adhesion of stimulated platelets to neutrophils. Blood 1990;75:550-554.

11. von Hundelshausen P, Koenen RR, Sack M, Mause SF, Adriaens W, Proudfoot AE, Hackeng TM, Weber C. Heterophilic interactions of platelet factor 4 and RANTES promote monocyte arrest on endothelium. Blood 2005;105:924-930.

12. Zarbock A, Singbartl K, Ley K. Complete reversal of acid-induced acute lung injury by blocking of platelet-neutrophil aggregation. J Clin Invest 2006;116:3211-3219.

13. Reutershan J, Basit A, Galkina EV, Ley K. Sequential recruitment of neutrophils into lung and bronchoalveolar lavage fluid in LPS-induced acute lung injury. Am J Physiol Lung Cell Mol Physiol 2005;289:L807-L815.

14. Koenen RR, von Hundelshausen P, Nesmelova IV, Zernecke A, Liehn EA, Sarabi A, Kramp BK, Piccinini AM, Paludan SR, Kowalska MA et al. Disrupting functional interactions between platelet chemokines inhibits atherosclerosis in hyperlipidemic mice. Nat Med 2009;15:97-103.

15. Matute-Bello G, Downey G, Moore BB, Groshong SD, Matthay MA, Slutsky AS, Kuebler WM; Acute Lung Injury in Animals Study Group. An official American Thoracic Society workshop report: features and measurements of experimental acute lung injury in animals. Am J Respir Cell Mol Biol 2011;44:725-738.

16. Tabuchi A, Mertens M, Kuppe H, Pries AR, Kuebler WM. Intravital microscopy of the murine pulmonary microcirculation. J Appl Physiol 2008;104:338-346.
17. Drechsler M, Megens RT, van Zandvoort M, Weber C, Soehnlein O. Hyperlipidemia-triggered neutrophilia promotes early atherosclerosis. Circulation 2010;122:1837-1845.

18. Soehnlein O, Kai-Larsen Y, Frithiof R, Sorensen OE, Kenne E, Scharffetter-Kochanek K, Eriksson EE, Herwald H, Agerberth B, Lindbom L. Neutrophil primary granule proteins HBP and HNP1-3 boost bacterial phagocytosis by human and murine macrophages. J Clin Invest 2008;118:3491-3502.

19. Clark SR, Ma AC, Tavener SA, McDonald B, Goodarzi Z, Kelly MM, Patel KD, Chakrabarti S, McAvoy E, Sinclair GD, et al. Platelet TLR4 activates neutrophil extracellular traps to ensnare bacteria in septic blood. Nat Med 2007;13:463-469.

20. Oehmcke S, Mörgelin M, Herwald H. Activation of the human contact system on neutrophil extracellular traps. J Innate Immun 2009;1:225-230.

21. Makino Y, Cook DN, Smithies O, Hwang OY, Neilson EG, Turka LA, Sato H, Wells AD, Danoff TM. Impaired T cell function in RANTESdeficient mice. Clin Immunol 2002;102:302-329.

22. Tyner JW, Uchida O, Kajiwara N, Kim EY, Patel AC, O'Sullivan MP, Walter MJ, Schwendener RA, Cook DN, Danoff TM, et al. CCL5CCR5 interaction provides antiapoptotic signals for macrophage survival during viral infection. Nat Med 2005;11:1180-1187.

23. Ley K, Laudanna C, Cybulsky MI, Nourshargh S. Getting to the site of inflammation: the leukocyte adhesion cascade updated. Nat Rev Immunol 2007;7:678-689.

24. Doerschuk CM. Mechanisms of leukocyte sequestration in inflamed lungs. Microcirculation 2001;8:71-88.

25. Burns JA, Issekutz TB, Yagita H, Issekutz AC. The alpha 4 beta 1 (very late antigen (VLA)-4, CD49d/CD29) and alpha 5 beta 1 (VLA-5, CD49e/ CD29) integrins mediate beta 2 (CD11/CD18) integrin-independent neutrophil recruitment to endotoxin-induced lung inflammation. J Immunol 2001;166:4644-4649.

26. Folkesson HG, Matthay MA. Inhibition of CD18 or CD11b attenuates acute lung injury after acid instillation in rabbits. J Appl Physiol 1997; $82: 1743-1750$

27. Doerschuk CM, Tasaka S, Wang Q. CD11/CD18-dependent and -independent neutrophil emigration in the lungs: how do neutrophils know which route to take? Am J Respir Cell Mol Biol 2000;23:133-136.

28. Kreisel D, Nava RG, Li W, Zinselmeyer BH, Wang B, Lai J, Pless R, Gelman AE, Krupnick AS, Miller MJ. In vivo two-photon imaging reveals monocyte-dependent neutrophil extravasation during pulmonary inflammation. Proc Natl Acad Sci U S A 2010;107:18073-18078.

29. Kornerup KN, Salmon GP, Pitchford SC, Liu WL, Page CP. Circulating platelet-neutrophil complexes are important for subsequent neutrophil activation and migration. J Appl Physiol 2010;109:758-767.

30. Singbartl K, Forlow SB, Ley K. Platelet, but not endothelial, P-selectin is critical for neutrophil-mediated acute postischemic renal failure. FASEB J 2001;15:2337-2344

31. Zarbock A, Polanowska-Grabowska RK, Ley K. Platelet-neutrophilinteractions: linking hemostasis and inflammation. Blood Rev 2007;21: 99-111.

32. Flad HD, Brandt E. Platelet-derived chemokines: pathophysiology and therapeutic aspects. Cell Mol Life Sci 2010;67:2363-2386.

33. Gerard C, Frossard JL, Bhatia M, Saluja A, Gerard NP, Lu B, Steer M. Targeted disruption of the beta-chemokine receptor CCR1 protects against pancreatitis-associated lung injury. J Clin Invest 1997;100:2022-2027.

34. Bhatia M, Proudfoot AE, Wells TN, Christmas S, Neoptolemos JP, Slavin J. Treatment with Met-RANTES reduces lung injury in caerulein-induced pancreatitis. Br J Surg 2003;90:698-704.

35. Nie L, Xiang R, Zhou W, Lu B, Cheng D, Gao J. Attenuation of acute lung inflammation induced by cigarette smoke in CXCR3 knockout mice. Respir Res 2008;9:82.

36. Pan ZZ, Parkyn L, Ray A, Ray P. Inducible lung-specific expression of RANTES: preferential recruitment of neutrophils. Am J Physiol Lung Cell Mol Physiol 2000;279:L658-L666.

37. Petersen F, Ludwig A, Flad HD, Brandt E. TNF-alpha renders human neutrophils responsive to platelet factor 4. Comparison of PF-4 and IL-8 reveals different activity profiles of the two chemokines. I Immunol 1996;156:1954-1962.

38. Baltus T, von Hundelshausen P, Mause SF, Buhre W, Rossaint R, Weber C. Differential and additive effects of platelet-derived chemokines on monocyte arrest on inflamed endothelium under flow conditions. J Leukoc Biol 2005;78:435-441. 